\title{
Enfermagem, uma ecologia de saberes
}

Enfermería, una ecología de los saberes

Nursing, an ecology of knowledges

Paulo Joaquim Pina Queirós

PhD, Professor Coordenador, Escola Superior de Enfermagem de Coimbra - Portugal.

Cómo citar este artículo en edición digital: Pina Queirós, P.J. (2016). Enfermagem, uma ecologia de saberes. Cultura de los Cuidados (Edición digital), 20(45). Disponible en: < http://dx.doi.org/10.14198/cuid.2016.45.15>

\author{
Correspondencia: Remitirse al correo electrónico \\ Correo electrónico: pauloqueiros@esenfc.pt, \\ Recibido: 2/10//2015; Aceptado: 10/06/2016
}

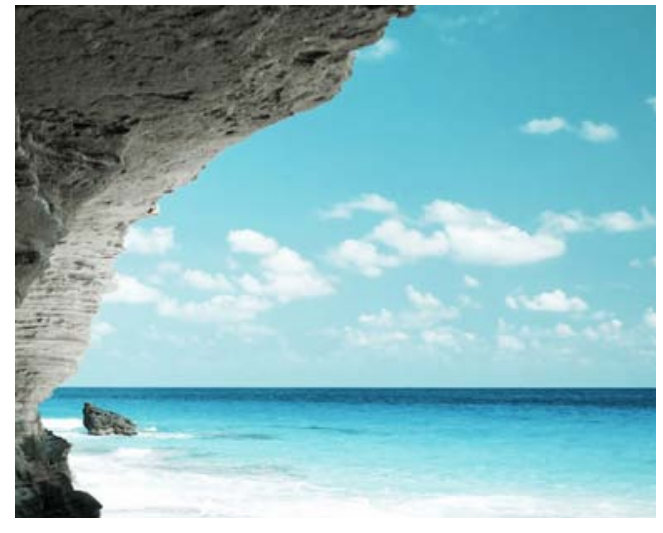

\section{ABSTRACT}

Objective: Contribute to situate and characterize the knowledge in nursing.

Methodology: Original theoretical reflection. Development: The notion of nursing as an ecology of knowledge calls for a clarification of the status of science that we assign to it. Nursing affirms itself as science in a context of paradigmatic transition, which, on the one hand, creates some difficulties, but, on the other hand, opens a wide range of opportunities. Nursing is action knowledge expressed in professionalized integral care, thus it is a practical human science. It is a science of plural knowledge.

Conclusion: The specific knowledge of nursing results from a process situated in a given context, where, in a process of reflection in action in a hermeneutic spiral, scientific knowledge intersects with other experiential knowledge. A mix of knowledge that corresponds to the various patterns. These patterns coexist and interact with each other and the context, which turns nursing knowledge into a true ecology of knowledge. The systematization of nursing knowledge will be truly promising in the light of an epistemological pragmatism and an understanding of the operations of systematization that allow moving from personal knowledge to public knowledge. Public knowledge is systematized and validated to become scientific knowledge.

Keywords: Nursing; Nursing care; knowledge; Ecology of knowledge

\section{RESUMEN}

Objectivo: Localizar y caracterizar el conocimiento en enfermería.

Método: Reflexión teórica original. Desarrollo: Considerar la enfermería como una ecología de saberes nos lleva a la aclaración del estatuto de ciencia que le atribuimos. La enfermería se afirma como ciencia en un marco de transición paradigmática, lo que, por un lado, determina algunas dificultades pero, por otro, abre una inmensidad de oportunidades. La enfermería es un conocimiento de acción y, por eso, una ciencia humana práctica. Acción que se expresa en el cuidado integral profesionalizado. Se trata de una ciencia de saberes plurales. 
Conclusiones: El conocimiento específico de enfermería resulta de un proceso situado en un contexto donde, en espiral hermenéutica, en un proceso de reflexión en la acción, se entrecruzan saberes científicos con otros saberes. Una mezcla de conocimientos que corresponden a varios patrones. Estos coexisten y actúan entre sí, y entre sí y el contexto, lo que hace que el conocimiento en enfermería sea una verdadera ecología de saberes. La sistematización del conocimiento en enfermería será verdaderamente prometedora a la luz de un pragmatismo epistemológico, percibiendo las operaciones de sistematización que permiten pasar del conocimiento personal al conocimiento público. Conocimiento público, sistematizado y validado, convertido en conocimiento científico.

Palabras clave: Enfermería; Cuidados de enfermería; Conocimiento; Ecología de los saberes.

\section{RESUMO}

Objectivo: Contribuir para situar e caracterizar o conhecimento em enfermagem.

Método: Reflexão teórica original.

Desenvolvimento: Considerar a enfermagem como uma ecologia de saberes remete-nos para a clarificação do estatuto de ciência que lhe atribuímos. A Enfermagem afirma-se como ciência, num quadro de transição paradigmática, o que determina por um lado algumas dificuldades, mas abre por outro uma imensidão de oportunidades. A Enfermagem é um conhecimento de ação, por isso uma ciência humana prática. Ação que se expressa no cuidar integral profissionalizado. E é uma ciência de saberes plurais.

Conclusão: O conhecimento específico de enfermagem resulta de um processo situado, num contexto, onde em espiral hermenêutica, num processo de reflexão na ação, se entrecru- zam saberes científicos com outros saberes. Um misto de conhecimentos que correspondem a vários padrões. Estes coexistem e agem entre si, e entre si e o contexto, o que faz do conhecimento em enfermagem uma verdadeira ecologia de saberes. A sistematização do conhecimento em enfermagem será verdadeiramente promissora à luz de um pragmatismo epistemológico, percebendo as operações de sistematização que permitem passar do conhecimento pessoal ao conhecimento público. Conhecimento público, sistematizado e validado tornado conhecimento científico.

Palavras chave: Enfermagem; Cuidados de Enfermagem; Conhecimento;

\section{INTRODUÇÃO}

Considerar a Enfermagem como uma ecologia de saberes remete-nos para a clarificação do estatuto de ciência que atribuímos à enfermagem, mas que nem todos reconhecem. Os termos ecologia e saberes, saber no plural, transporta-nos para o universo da pluralidade de conhecimentos, que interessam à enfermagem e a ela estão ligados. Por outro lado, a noção de ecologia, que na estrita definição biológica reporta-se à "ciência que estuda as relações dos seres vivos entre si ou com o meio orgânico ou inorgânico onde vivem" (Houaiss, Vilar, \& Franco 2003, p.1422), pode ser usada analogicamente, para nos indicar as relações reciprocas entre as diversas formas de saber, entre si e o meio onde ocorrem, um contexto específico, situado, concreto. Relevando do termo ecologia as dimensões: relação reciproca, equilíbrio dinâmico entre partes, e das partes com um meio, um ambiente ou seja um contexto.

Colocamos como objetivo deste trabalho: Contribuir para situar e caracterizar o conhe- 
cimento em enfermagem, e clarificar o estatuto de ciência que lhe atribuímos. O que pensamos possível através do método de reflexão teórica original, tendo como ponto de partida a bibliografia oportuna.

Importa clarificar que a noção ecologia de saberes que aqui trazemos tem uma filiação teórica epistemológica, com um propósito definido à partida, o de contribuir para discussão do que é a enfermagem, que conhecimento utiliza na sua ação, que saberes cria e sistematiza, como se define como ciência, como se situa no contexto disciplinar no quadro geral das ciências.

A noção ecologia de saberes que nos interessa, é a reportada por Sousa Santos, a "propósito da inesgotável diversidade da experiência do mundo" (Santos, 2007a, p.23), num quadro de evolução das ciências, enquanto construções humanas, onde "todo o conhecimento é contextual” (Santos, 2002, p.86), e o conhecimento científico tem uma dupla contextualização, pela comunidade científica e pela sociedade, o que implica ser simultaneamente uma prática científica e uma prática social (Santos, 2002).

Este autor, socorrendo-se de um modelo interpretativo Kuhneano, descreve a fase de desenvolvimento atual do conhecimento como de transição paradigmática, onde as ciências, após o apogeu de afirmação da modernidade, deram lugar a uma pós-modernidade, ela mesma não estática, evolutiva, em que se distinguem, o pós-modernismo celebratório, de continuidade defensiva, e o pós-modernismo de oposição. Deixando antever a possibilidade de um pensamento, já não só pós-moderno, mas pós-abissal, num quadro de uma epistemologia pragmática, resgatada à epistemologia clássica (Nunes, 2008). É neste quadro teórico que a ecologia de saberes surge como um "pensamento alternativo de alternativas", metaforicamente explicitada como uma "epistemologia do Sul", em que importa também, no âmbito da "sociologia das ausências", a "ideia de que muito daquilo que não existe é produzido ativamente como não existindo" (Santos, 2007b, p.178).

A ecologia de saberes, diz-nos Nunes (2008, p.62) citando Santos (2007a) "não concebe os conhecimentos em abstrato, mas antes como práticas de conhecimento que possibilitam ou impedem certas intervenções no mundo real", e ainda que "a epistemologia passa a abranger explicitamente todos os saberes - deixando de os tratar apenas através da sua relação com os saberes científicos" (Nunes, 2008, p.47). "Reconhecer a validade e dignidade de todos os saberes implica que nenhum saber poderá ser desqualificado antes de ter sido posto à prova a sua pertinência e validade em condições situadas" (Nunes, 2008, p.62). Afirmando ainda "as hierarquias de saberes não podem ser definidas a partir da soberania epistémica de um modo de saber ..., mas de forma pragmática, isto é, indissociável das práticas situadas de produção de saberes." (Nunes, 2008, p.66).

De onde se percebe que "o reconhecimento da diversidade das formas de conhecer - uma diversidade cujos limites são impossíveis de estabelecer previamente ao envolvimento ativo com essas formas - obriga a redefinir as condições de emergência, de desenvolvimento e de validade de cada uma das formas, incluindo a ciência moderna, que passa assim a ser objeto de uma avaliação situada que obriga à "simetrização" radical de todos os saberes" (Nunes, 2008, p.62-63). "Como ecologia de saberes, o pensamento pós-abissal tem como premissa a ideia da diversidade epistemológica do mundo, o reconhecimento da existência de uma pluralidade de formas de conhecimen- 


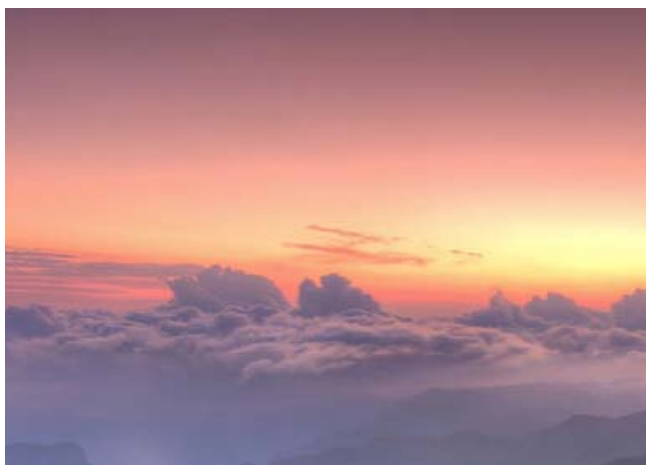

to além do conhecimento científico" (Santos, 2007a, p.23), que confronta a "monocultura da ciência moderna com uma ecologia de saberes" (Santos, 2007a, p.22). De forma expressiva Santos (2003) considera o conhecimento científico moderno "um conhecimento mínimo que fecha as portas a muitos outros saberes..." (Santos, 2003, p.32).

Do conceito de ecologia de saberes de Boaventura Sousa Santos realça-se, a multiplicidade de saberes não hierarquizados, e a validação do conhecimento(s) pela ação, conhecimentos situados. Rompendo com uma "racionalidade indolente", contribuindo para a criação de um novo "senso comum" (Santos, 2007b), onde a ciência moderna, pós-abissal, seja parte integrante e não dominante de uma ecologia de saberes.

\section{DESENVOLVIMENTO DO TEMA}

Num quadro de transição paradigmática, temos uma enfermagem a afirmar-se como ciência, a conquistar espaço e estatuto, à procura da sua legitimidade epistémica, em que "a ciência em geral e não apenas as ciências sociais se pautam por um paradigma epistemológico e um modelo de racionalidade que davam [dão] sinais de exaustão" (Santos, 2004, p.1). O que determina por um lado algumas dificuldades, mas abre por outro uma imensidão de oportunidades. Neste contexto a enfermagem defronta-se com um quadro caracterizado por: i) Domínio de uma racionalidade positivista, subalternizando todas as outras formas de ciência considerando-as secundárias, quando “... não há uma maneira única de produzir ciência. A própria ciência é plural internamente" (Santos, 2007b, p.185); ii) Relegar para segundo plano, outras formas de ciência que não as naturais. Permitindo, com condescendência o estatuto de ciência às ciências sociais; iii) Reconhecimento de outras formas de conhecimento como sejam a filosofia e a teologia, mas de outra ordem, que não ciência; iv) Reconhecimento das humanidades como formas de conhecimento, mas de outra grandeza que não ciência. Desta forma as leituras do real estão necessariamente afuniladas e restringidas ao conhecimento científico, pelo critério de validade único, desvalorizando qualquer outra racionalidade, num processo descrito por $\mathrm{Nu}$ nes (2008) como epistemicídio.

Para Santos (2004, p.2), a modernidade conduziu à "separação absoluta entre conhecimento científico - considerado único válido e rigoroso - e outras formas de conhecimento como o senso comum ou estudos humanísticos”. De onde decorre, que a ciência moderna é bem marcada e entrincheirada, num corte abissal com as outras leituras do real, sendo que nesta perspetiva ciência valida é a que distingue claramente sujeito do objeto, reduz a complexidade do real a leis simples suscetíveis de formulação matemática, a que resulta de um mecanicismo determinista e de uma causalidade funcional (Santos, 2004). Importa referir que no âmbito de uma outra abordagem, o pensamento pós-abissal, apresentado por Boaventura Santos, "o que está em causa não é desprezar a ciência, é coloca-la no seu contexto". Para prosseguir afirmando: "É o monopólio do rigor que nós 
criticamos. E, portanto, estamos em condições de poder apreciar o que na ciência deve ser apreciado, e deve ser resgatado ao mesmo tempo criando espaços para outros conhecimentos, para outras experiências de saberes" (Santos, 2007b, p.178). É que se não houver uma "epistemologia ampla" perde-se muito do saber. Saberes perdidos, numa produção de ausências, caracterizada por cinco grandes formas: Monocultura do saber cientifico e do rigor, dando origem a uma forma especifica de ausência: o ignorante. Monocultura das classificações, assente em hierarquizações, traduzindo-se em considerar algo ou alguém inferior. Monocultura da escala dominante, onde prevalece o universal e o global em detrimento do local. Monocultura do tempo linear, ocultando a natural dessincronização e a assimetria. Monocultura da produção capitalista, concentrando a produtividade num ciclo único levado à exaustão, e ao seu esgotamento, por oposição à renovação natural dos ciclos. (Santos, 2007b).

O paradigma da modernidade foi fundamental para o desenvolvimento humano, nele a ciência conforme a conhecemos e com as características que atrás descrevemos. Com a técnica e a tecnologia disponibilizada, foram e são essenciais à criação de sociedades de bem-estar, embora de forma manifestamente desigual e assimétrica. "O modelo de racionalidade que preside à ciência moderna constitui-se a partir da revolução científica do século XVI e foi desenvolvido nos séculos seguintes basicamente no domínio das ciências naturais." (Santos, 2000, p.58). É a partir daqui, que "pode falar-se de um modelo global (isto é, ocidental) da racionalidade científica que admite variabilidade interna, mas que se defende ostensivamente de duas formas de conhecimento não científico (e, portanto, potencialmente pertur- badoras): o senso comum e as chamadas humanidades ou estudos humanísticos..." (Santos, 2000, p.58). O modelo deste paradigma é o das ciências da natureza, exatas, assentes numa racionalidade científica, cognitiva-instrumental da ciência e da tecnologia, que se foi impondo a outras formas de racionalidade como sejam a moral-prática e a estética-expressiva (Santos, 2000). Até as ciências sociais quando surgem, surgem como ciências à imagem das ciências exactas, por exemplo, a sociologia surge como física social com Comte, a análise de suicídio de Durkheim assente em os métodos matemáticos. Só tardiamente as ciências sociais assumem autonomia diferenciada com metodologias próprias. Também nas ciências médicas, o positivismo impôs-se onde foi de grande relevância o trabalho de Claude Bernard, impondo meritoriamente, o rigor científico, bem expresso na obra "Introdução ao estudo da medicina experimental" publicada em 1865. Esta obra "descreve o método científico com bastante finura e cambiantes, muito mais do que na maior parte dos atuais manuais de ciências que, no entanto, se referem ao seu esquema." (Fourez, 2008, p.33).

Pioneiro na introdução da medicina moderna em Portugal, Costa Simões, "verdadeiro fundador da primeira escola experimental em Portugal” (Salgado, 2003, p.31), realizou duas viagens científicas à europa. A primeira, contemporânea de Bernard, entre 1864 e 1865. Esta visita, segundo Salgado, biógrafo de Costa Simões, decorreu "ouvindo, observando e trabalhando com os mestres mais célebres daqueles países nos seus laboratórios" (Salgado, 2003, p.30). Da segunda viagem, em 1878, refere-nos Sobral Cid, na "Oração de Sapiência”, proferida na Universidade de Coimbra em 1907: "Na realidade, a nova era da Faculdade de medicina data de 1886, [resulta] da longa e 
curiosa viagem scientifica que o emérito professor dr. António Augusto da Costa Simões, o primeiro fundador intelectual da Faculdade Moderna, realizou ...” (Salgado, 2003, p.31). Costa Simões, do que observa nas suas viagens, percebe a necessidade da qualificação dos profissionais que trabalham nos hospitais portugueses, e rápido, chega à conclusão da necessidade da formação estruturada dos enfermeiros, cria a primeira escola de enfermeiros em Portugal, precisamente em Coimbra, da qual a Escola Superior de Enfermagem de Coimbra é hoje herdeira, pondo em funcionamento o primeiro curso de enfermagem em Portugal, a 17 de outubro de 1881.

A enfermagem, como corpo de conhecimentos específicos, afirma-se como ciência humana com uma forte componente de ação. É caracterizada por Meleis como uma "ciência humana" e como sendo "uma disciplina orientada para a prática” (Meleis, 2012). Strasser (1985) e Kim (2010) definem enfermagem como uma "ciência humana prática".

O desenvolvimento do conhecimento e da teoria de enfermagem, nesta perspetiva da disciplina orientada para a prática, deve ir para além do progresso do conhecimento em si mesmo e fornecer uma base sólida que ajude a conhecer as melhores formas de cuidar das pessoas, famílias e comunidades. A natureza da enfermagem enquanto disciplina do conhecimento não se esgota na sua dimensão prática. Na realidade, faz todo o sentido, como se disse, perspetivar a enfermagem enquanto uma "ciência humana prática” (Meleis, 2005, p.94). É próprio das ciências humanas práticas serem baseadas na ação comunicativa e pressuporem a participação direta em algum tipo de intervenção (Medina, 1999). As ciências humanas práticas diferenciam-se, das ciências humanas teóricas e das ciências humanas teóricas com aspetos práticos, pelo estilo de comunicação e finalidade (Strasser,1985). É comum ao conjunto de ciências humanas: poder comunicar e compartilhar significados com os sujeitos que estudam; o seu papel não ser meramente explicativo mas sobretudo de compreensão; e ainda os seus membros considerarem-se a si mesmo como parte integrante do mundo que pretendem estudar e compreender (Strasser, 1985; Medina 1999).

Na ciência de enfermagem o conhecimento criado e utilizado encontra-se fortemente imbuído de prática, estruturando-se num processo que é definido como "círculo hermenêutico" (Gadamer,1998) e explicitado por Bishop e Scudder (1995) como "espiral hermenêutica". Neste processo de criação e sistematização do saber, o conhecimento, ocorre na ligação constante e recursiva, prática-teoria/teoria-prática, naquilo que é designado por "movimento de translação de conhecimento", como seja o processo de converter descobertas científicas em benefícios para a saúde (Sobrinho-Simões, 2012). A natureza do conhecimento criado na ação, na clínica, identifica-se com as respostas que os profissionais encontram para as exigências e, problemas, com que constantemente são confrontados, próprios das situações de saúde e de doença. Situações quase sempre complexas, e fazendo com que os cuidados às pessoas sejam igualmente complexos (Bastos, 2012). Este conhecimento criado na ação, ocorre num palco de incerteza, vulnerabilidade e grande variabilidade. Desenvolve-se em uma realidade complexa, incerta e saturada de valores (Medina-Moya, 2008). O saber criado, originado nestas respostas, corresponde a operações mentais no quadro de uma racionalidade designada por prático-reflexiva. Neste quadro estão presentes operações como reflexão na ação, reflexão sobre a ação e reflexão sobre 
a reflexão na ação (Alarcão, 2001; Alarcão \& Tavares, 2003; Schön, 1992).

"Para Schön, o profissional não atua seguindo a lógica instrumental da racionalidade técnica. Antes pelo contrário, o profissional é um prático reflexivo cuja ação se funda num conhecimento prático e tácito que se ativa durante a ação... o elemento fundamental aqui é a reflexão em ação, posto que é o mecanismo mediante o qual se gera o conhecimento profissional (prático) que habilita para o exercício competente do cuidado." (Moya, 2005, p.488). A ação clínica é algo só possível num determinado ambiente, concretizada numa determinada prática, num sistema ecológico concreto.

A enfermagem é uma ciência de saberes plurais. O processo de desenvolvimento de conhecimento específico em enfermagem, assenta numa multiplicidade de padrões, inicialmente identificados no âmbito do saber em enfermagem, como padrão empírico, estético, ético, pessoal (Carper,1978), mas também posteriormente acrescidos de padrões reflexivo e sociopolítico (Carper, 1992; Carper, 2006). A estes padrões, outros autores acrescentam outras formulações, enfatizando outros saberes incorporados, como sejam cultural, tácito, intuitivo, processual. Percebe-se como refere Schön (1992) a necessidade de criar ou revitalizar uma fenomenologia da prática que inclua, com centralidade na reflexão sobre a reflexão-na-ação dos profissionais em seus ambientes organizacionais.

Os padrões de conhecimento agrupam formas de saber que se interligam e se relacionam com as evidências científicas, a sensibilidade e a forma pessoal de cuidar harmoniosamente, a ponderação ética, a utilização da capacidade relacional, das características próprias da personalidade, da constante reflexão, e no conhecimento dos contextos. Estas formas de saber desenvolvem-se, recriam-se e treinam-se, na caminhada de desenvolvimento de competências, desde iniciado a perito (Benner, 2001).

Estas diferentes formas de conhecimento estão na prática clínica, nas intervenções de enfermagem, constituindo-se como conhecimento privado, do domínio de cada um, que ao ser sistematizado e validado se torna em conhecimento público (Kim, 2010). Conhecimento de padrões diversificados, interligados, não hierarquizados, numa "ecologia de saberes" própria de uma epistemologia, já não simplesmente considerada como "pós-moderna", superada a dicotomia ciências exatas/ciências humanas e sociais, mas sobretudo identificada como "pós-abissal" (Santos, 2007a; Nunes, 2008), no sentido que para a compreensão integral da dimensão humana importa outros saberes para além do conhecimento científico.

A enfermagem é assim uma ciência humana prática, cujo saber, fruto de uma racionalidade prático-reflexiva, resultante da clínica, ao ser sistematizado se constitui em saber próprio disciplinar. Competências que se desenvolvem fruto da "existência de um conhecimento implícito que se ativa com a ação da enfermeira e que permite o reconhecimento instantâneo de situações problemáticas e respostas intuitivas" (Moya, 2005, p.489).

A sistematização do conhecimento específico é um processo contínuo onde se vão estruturando conceitos (linguagem própria disciplinar) e teorias específicas (grandes teorias, teorias de médio alcance, teorias situacionais). A riqueza conceptual e teórica disciplinar está bem patente na diversidade de conceitos próprios e conceitos migratórios com significado próprio na disciplina de enfermagem. E ainda nas dezenas de teorias de enfermagem que permitem leituras enquadradoras de práticas 
clínicas tão diversificadas. É num quadro diversificado de conceitos, teorias e modelos que assenta a riqueza conceptual da ciência de enfermagem, correspondendo à construção teórica, do conhecimento utilizado e surgido na prática clínica. A disciplina de Enfermagem tem definido os seus conceitos metaparadigmáticos: Pessoa, Saúde, Ambiente e Enfermagem, apresentados por Yura e Torres (1975) e desenvolvidos por Fawcett (1991). E o seu domínio, considerado como: "a perspetiva e o território da disciplina. Inclui o objeto de interesse, os valores e crenças consensuais, os conceitos centrais, os fenómenos de interesse, os principais problemas, e os métodos utilizados no fornecimento de respostas" (Meleis, 2005, p. 11). Metaforicamente, o domínio da disciplina de enfermagem corresponde ao seu $\mathrm{ADN}$, àquilo que lhe confere identidade e singularidade. É ainda com Meleis, que podemos identificar sete conceitos centrais, intimamente relacionados, que caracterizam o domínio da enfermagem: pessoa/cliente, transições, interação, ambiente, processo de enfermagem, intervenções de enfermagem e saúde.

A pluralidade de conhecimentos, saberes que são colocados em ação no processo de cuidar, cuidar integral profissionalizado, são de natureza plural, estruturam-se numa interligação entre conhecimento investigado e sistematizado - o conhecimento público, e os saberes da experiência, da intuição, da sensibilidade, da resposta imediata e altamente contextualizada - o conhecimento privado. Operação que só é possível quando entendida como conhecimento que surge da ação, por isso situado, contextualizado, que pode ser sistematizado e usufruir de sistematizações anteriores.

No âmbito da racionalidade prático-reflexiva, - não se trata da desvalorização do conhecimento científico, do padrão de conhecimento empírico, consubstanciado em evidências científicas que transportaremos para a prática, uma prática também baseada em evidencias que não desprezamos - , mas antes em não menosprezar outras formas de saber, tão importantes e úteis para o cuidar integral. A este propósito vale a pena lembrar Benner, Tanner e Chelsa (2009), quando alertam que as disciplinas sofrem com a obscuridade do conhecimento prático ou clínico já que o conhecimento científico formal e a tomada de decisão técnica racional tendem a ser as únicas formas legítimas de conhecimento. Por outro lado, reduzir o profissional ao técnico é retirar todo o sentido à prestação de cuidados, aos cuidados, palco de construção de saberes, onde os profissionais constroem conhecimento a partir da reflexão sobre as suas práticas, numa epistemologia da prática (Schön, 1992).

\section{CONCLUSÕES}

Encaramos a enfermagem como disciplina científica, onde os saberes de natureza empírica se articulam com outros saberes de igual valia, onde uma componente metodológica forte opera na sistematização do conhecimento privado a interagir numa espiral hermenêutica, com as evidências já conhecidas, contextualizada na ação clínica, localizada num ambiente, criando e recreando novo conhecimento.

Realça-se desta forma, o poder da clínica, num âmbito de uma racionalidade prático-reflexiva. Este conhecimento clínico mostra-se pelo reconhecimento de padrões, compreensão das mudanças na resposta humana ao longo do tempo, na capacidade de fazer distinções qualitativas, num reconhecimento muitas vezes tácito, na identificação de subtis mudanças. O enfermeiro é um "profissional prático-refle- 
xivo cuja ação se baseia num conhecimento prático e tácito que se ativa durante a ação e no qual podem, sobre tudo a efeitos heurísticos, distinguir-se três componentes: conhecimento em ação, reflexão em ação e reflexão sobre a reflexão em ação" (Moya, 2005, p.488). $O$ cuidado de enfermagem, resultante da ação, é um “momento contínuo de reflexão onde o ser percebe-se em sua incompletude e lança-se em uma dinâmica de realização das necessidades do outro em suas inúmeras possibilidades" (Kempfer \& Carraro, 2012, p.79). Onde o sentido competente, de peritagem, deriva de uma compreensão global da situação, da valoração holística e qualitativa, que passa por três características: esquemas de reconhecimento (padrões); sentido de proeminência (destaque) e consciência situacional. "A perita, que tem uma enorme experiência, compreende, agora, de maneira intuitiva cada situação e apreende diretamente o problema sem se perder num largo leque de soluções e de diagnósticos estéreis" (Benner, 2001, p.58). "A enfermeira perita é capaz de reconhecer o que é notável em determinadas situações" (Benner, Tanner \& Catherine, 2009, p.347), “... apercebe-se da situação como um todo, utiliza como paradigmas de base situações concretas que ela já viveu e vai diretamente ao centro do problema sem ter em conta um grande número de considerações inúteis" (Dreyfus, H, 1979; Dreyfus, S,1981, in Benner, 2001, p.33).

Em algumas áreas da enfermagem existe uma forte incorporação de técnicas, que requerem destreza e treino. As técnicas, pela diferenciação que comportam e segurança que permitem, têm forte atratividade, tornam os especialistas em verdadeiros técnicos, aplicadores de elevado desempenho, numa racionalidade técnica, em que o padrão de conhecimento empírico se torna dominante em detrimento de outras formas de saber. A obsessão pela prática baseada em evidências, desvirtua a clínica, também baseado no conhecimento estético, ético, processual, tácito, de contexto. A Enfermagem afirma-se numa ecologia de saberes, saberes científicos, e saberes de outra natureza, contextualizados, situados, ecologicamente considerados, a contribuir para uma ação e resultantes dessa acção, permitindo o cuidar integral profissionalizado. Cuidar como acção - prestação de cuidados, e atitude de compaixão, de solicitude, de disponibilidade. Cuidar integral da pessoa e do ambiente. Cuidar organizado, ensinado, estruturado e intencional ou seja profissionalizado.

\section{REFERÊNCIAS}

- Alarcão, I. (2001). Escola reflexiva e nova racionalidade. Porto Alegre: Artmed Editora.

- Alarcão, I., Tavares, J. (2003). Supervisão da prática pedagógica. Uma perspetiva de desenvolvimento e aprendizagem. $2^{\circ}$ ed. Coimbra: Editora Almedina.

- Basto, M. L. (2012). Cuidar em enfermagem. Saberes da prática. Coimbra: Formasau Lda

- Benner, P. (2001). De Iniciado a Perito: Excelência do Poder na Prática Clínica de Enfermagem. Coimbra: Quarteto Editora.

- Benner, P., Tanner, C., \& Chelsa, C. (2009): Expertise in nursing practice: caring, clinical judgment \& ethios. $2^{\text {nd }}$ ed. New York: Springer Publishing Company, LLC.

- Bishop, A., \& Scudder, J. (1995). Applied Science, Practice and Intervention Tecnology. In A. Omery, C. Karper, G. Page (Eds), In Search of nursing Science (pp.263-274). London: Sage Publications.

- Carper, B. (1978). Fundamental Patterns of knowing in Nursing. Advances in Nursing Science, 1(1), 13-24.

- Carper, B. (1992): Philosophical inquiry in nursing: an application. In Kikuchi, J.F, Simmons H. (eds). Philosophical Inquiry in nursing. Newbury Parck CA: Sage.

- Carper, B. (2006). Fundamental patterns of knowing in nursing. In L. C. Andrist, P. K. Nicholas \& K. A. Wolf (Eds.), A history of nursing ideas (pp. 129-137). Sudbury, Canada: Jones and Bartlett Publishers.

- Fawcett, J. (1991). Aproaches to knowledge developmet in nursing. Can J Nurs Res. 23(4), 23-33.

- Fourez, G. (2008). A construção das ciências. As lógicas das invenções científicas. Lisboa: Instituto Piaget. 
- Gadamer, H.G. (1998). Verdade e Método: traços fundamentais de uma hermenêutica filosófica. 2.ed. Petrópolis: Editora Vozes.

- Houaiss, A., Villar, M., \& Franco, F. (2003). Dicionário Houaiss da Língua Portuguesa. Lisboa: Círculo de Leitores.

- Kempfer, S.; Carraro, T. (2012): Ser-aluno de enfermagem e a experiência do cuidado: revisão integrativa. Cultura de los cuidados, 16 (33), 75-80.

- Kim, H. S. (2010): The nature of theorical thinking in nursing ( $3^{\circ} \mathrm{ed}$.). New York: Springer Publishing Company.

- Medina, J.L. (1999). La pedagogía del cuidado: saberes y práticas en la formación universitaria en enfermería. Barcelona: Laertes.

- Moya, J.L. (2005). Redescubrir el saber práctico de la enfermera. El difícil equilibrio académico y profesional en la españa "preeuropea« (III). Rev Enferm, 28 (7-8), 490.

- Medina-Moya, J.L. (2008). De mapas y territórios. For-malización de los saberes profesionales en el curriculum. Rev ROL Enferm, 31(7-8),533-6.

- Meleis, A. I. (2005) Theoretical Nursing: Development and Progress. Philadelphia: Lippincott Williams \& Wilkins.

- Meleis, A. I. (2012). Theorical nursing. Development and progress. $5^{\text {th }}$ ed. Philadelphia (US): Wolters Kluwer/Lippincott Williams \& Wilkins.

- Nunes, J.A.(2008). O resgate da epistemologia. Revista Crítica de Ciências Sociais, 80, 45-70.

- Santos, B. S. (2000). A crítica da razão indolente. Contra o derperdício da experiência. (Para um novo senso comum.
A ciência, o direito e a política na transição paradigmática. Vol. I). Porto: Edições Afrontamento.

- Santos, B. S. (2002). Introdução a uma ciência pósmoderna (6 ${ }^{\mathrm{a} e d}$.). Porto:Ed. Afrontamento.

- Santos, B. S. (2003). Um discurso sobre as ciências (14 ed.). Porto: Edições Afrontamento.

- Santos, B. S. (2004). Do pós-moderno ao pós-colonial. E para além de um e de outro. VIII Congresso LusoAfro-Brasileiro de Ciências Sociais (pp. 1-45). Coimbra: Centro de Estudos Sociais da Faculdade de Economia da Universidade de Coimbra.

- Santos, B. S. (2007a). Para além do pensamento abissal: Das linhas globais a uma ecologia de saberes. Revista Crítica de Ciências Sociais, 78,. 3-46.

- Santos, B. S. (2007b). Conhecimeto e transformação social: para uma ecologia dos saberes. Somanlu. Revista de Estudos Amazónicos, 1(7), 175-189.

- Schön, D. (1992). La formación de profesionales reflexives. Hacia um nuevo diseño dela formación y el aprendizaje en las profesiones. Madrid: Piadeos MEC. Ed.

- Sobrinho-Simões, M. (2012). Investigação em ciências da saúde: o papel da translação. Ata Med Port. Jan-Feb: 25(1),1-1.

- Strasser, S. (1985): Undertanding and explanation: Basic ideas concerning the humanity of the humans sciences. Pittsburg: Duquesne. University Press.

- Yura, H, \& Torres, G. (1975). Today's conceptual frameworks with the baccalaureate nursing programs (National League for Nursing Publication No. 15-1558, 17-75). New York: National League for Nursing. 